\title{
Consignas como herramientas mediadoras de lectura: análisis en materias de ciencias sociales del nivel secundario argentino
}

\author{
Natalia Rosli ${ }^{1}$ \\ Universidad de Buenos Aires, Argentina \\ nrosli85@gmail.com
}

\section{Resumen}

A la hora de analizar prácticas de enseñanza, es común el acuerdo entre investigadores acerca de la necesariedad de tomar en cuenta las interacciones entre las intervenciones docentes, las acciones de los alumnos y la especificidad del contenido disciplinar. Empero, no siempre los estudios consideran otros elementos que componen las situaciones didácticas, como las consignas y los materiales de lectura propuestos a los estudiantes que influyen en las maneras de comunicar, construir y aprender conocimientos en las aulas. El presente artículo considera la relevancia de analizar las consignas de lectura en tanto herramientas fundamentales que mediatizan la interacción de los alumnos con los textos (Aisenberg, 2005a, 2010).

Palabras clave: prácticas de enseñanza, situaciones didácticas, consignas, potencial educativo.

\section{Slogans as mediating tools for reading: analysis in social science subjects in the Argentinean secondary level}

\begin{abstract}
When analyzing teaching practices, it is common agreement among researches about the need to consider the interactions between teaching interventions, the actions of students and the specificity of the disciplinary content. However, research findings do not always consider other elements that compose didactic situations, such as slogans and reading materials proposed to students that influence the ways of communicating, building and learning knowledge in the classroom. This article considers the relevance of analyzing reading slogans as fundamental tools that mediate students' interaction with texts (Aisenberg, 2005a, 2010).
\end{abstract}

Key words: Teaching practices, didactic situations, slogans, educational potential.

1 Doctora en Ciencias de la Educación (Universidad Nacional de la Plata), Becaria posdoctoral (CONICET). Es integrante del equipo de investigación GICOLEM que dirige la Dra. Paula Carlino en el Instituto de Lingüística (UBA), quien además fue la directora de su tesis doctoral. Para más detalles de la labor investigativa del grupo: https://sites.google.com/site/giceolem2010/ 


\section{Introducción}

Partiendo de una concepción situada, sistémica y dinámica del funcionamiento didáctico (Rickenmann, 2006), entendemos como sistema didáctico al conjunto de interacciones entre tres componentes: alumnos, docentes y saberes (Chevallard, 1991). Las situaciones didácticas que los docentes proponen en las aulas implican otros elementos como tareas, consignas y materiales de lectura que suelen pasar desapercibidos en el análisis de las prácticas de enseñanza.

En relación con las consignas de lectura, existen trabajos que identifican distintos tipos en la escuela secundaria y la universidad. En el nivel universitario, Carlino (2005) distingue consignas que piden información localizable en el texto, en las que alcanza con subrayar una parte del texto, y consignas que exigen reconstruir ideas, en las que se necesita comprender el texto como un todo y/o vincular ideas. Para el nivel secundario, Dysthe (1996) diferencia dos tipos de consignas de lectura: preguntas que guían la revisión de puntos centrales de los libros de texto, que pueden resolverse directamente con la información allí disponible, y preguntas que no tienen respuestas prefijadas, por lo cual demandan a los estudiantes pensar y reflexionar por sí mismos.

Particularmente en la enseñanza de las Ciencias Sociales, Basuyau y Guyon (1994) identifican como predominantes las consignas de descomposición que requieren identificar y reproducir porciones del texto. Estudios didácticos en el nivel primario argentino muestran que este tipo de consignas originan en los estudiantes lecturas superficiales para localizar información puntual (Aisenberg, 2005a, 2010; Benchimol, Carabajal y Larramendy, 2008; Lerner, Aisenberg y Espinoza, 2009). Esta localización de respuestas se ve facilitada porque en las preguntas se reproducen frases textuales de los libros indicados para leer (Rockwell, 1995). Este quehacer lector de localizar información, que fomentaría este tipo de consignas, resulta imprescindible (Aisenberg, 2004) pero insuficiente para aprender contenidos a través de la lectura. Consignas de ese estilo permiten contestar las preguntas sin necesidad de entender el texto, a través de una correspondencia término a término entre las preguntas y los párrafos del material. Al respecto, Aisenberg, (2005b) comprobó a través de entrevistas de lectura a estudiantes del nivel primario que, tras leer un texto para localizar información, decían no haber entendido o daban explicaciones distantes de lo planteado.

Además de estas consignas de localización, existen otras consignas de lectura en la enseñanza de las Ciencias Sociales, que incitan a comprender el sentido del texto en su totalidad mediante la construcción de ideas globales, explicaciones o relaciones (Aisenberg, 2005a, 2010). Para ser contestadas, estas consignas requieren un conocimiento general del tema, por lo que suelen resultar complejas de entender para los estudiantes.

En línea con los resultados de los estudios citados, nos preguntamos qué tipo de consignas de lectura se proponen en materias de Ciencias Sociales de nivel secundario. Para responder este interrogante, este trabajo analiza las consignas propuestas en materias de una escuela secundaria argentina a la que asisten alumnos de sectores socioeconómicos desfavorecidos.

\section{Metodología}

Los datos presentados en este estudio corresponden a un trabajo de campo realizado en una institución secundaria de la Ciudad de Buenos Aires, Argentina. La escuela se localiza a pocas cuadras de un barrio con viviendas precarias, en el que habitan familias en situación de vulnerabilidad social. Particularmente, el curso de quinto año en el que indagamos está compuesto por 14 estudiantes: once mujeres y tres varones cuyas edades oscilan entre los 17 y 22 años. Hallamos que doce estudiantes desarrollaron trayectorias escolares discontinuas producto de repitencias (10) y/o abandonos (2), por lo que presentan sobreedad.

Se observaron 39 clases en tres materias de Ciencias Sociales (Estudios Sociales Argentinos, Geografía Económica y Legislación Fiscal), se 
entrevistó a sus docentes y a los alumnos. A su vez, se recolectaron documentos institucionales (proyecto institucional, documentos de trabajo confundamentos teórico-ideológicos), materiales de lectura (cuadernillo de textos, manuales y módulos escolares, artículos periodísticos, recortes de Internet), producciones escritas de los alumnos (carpetas, trabajos prácticos y evaluaciones).

Para una caracterización de las prácticas de enseñanza en dichas materias y un análisis del potencial educativo (Dysthe, 2013), esto es, las posibilidades de aprendizaje que crean y aquellas que cierran, remitimos al lector a la tesis doctoral de la autora (Rosli, 2016) y otros trabajos posteriores (Carlino y otros, en prensa; Rosli y Carlino, 2017, en prensa).

Específicamente, para este trabajo se rastrearon las consignas propuestas por los docentes a través de cuestionarios dictados oralmente, escritos en el pizarrón y/o en fotocopias que entregaban a los estudiantes, o que formaban parte de los materiales de lectura.

Una vez transcriptas y listadas las consignas en una tabla del programa Excel, efectuamos lecturas y relecturas de éstas junto con los materiales indicados para leer y responderlas. Comenzamos un proceso de categorización de la empiria seleccionada guiándonos por nuestro marco conceptual, que condujo a delimitar categorías de consignas. Luego, contabilizamos los ejemplos pertenecientes a cada categoría.

\section{Resultados}

Identificamos que en tres materias de Ciencias Sociales de un quinto año del nivel secundario, se plantean contestar cuestionarios con consignas globales y consignas de localización. A continuación, detallamos sus particularidades y analizamos los argumentos que proporcionan los docentes para su selección.

En Geografía Económica y Legislación Fiscal la mayoría de las preguntas pide identificar información puntual en los textos al igual que las consignas de descomposición descritas por
Basuyau y Guyon (1994). Para contestar estas consignas alcanza con localizar y extraer del texto las partes requeridas (Aisenberg, 2004, 2005a, 2010; Carlino, 2005). La Tabla 1 muestra cuatro ejemplos de estas consignas y resalta con subrayado la igualdad o similitud con párrafos de los materiales que los estudiantes tienen que leer en esas materias.

Tabla 1. Consignas de localización

\begin{tabular}{|c|c|}
\hline $\begin{array}{c}\text { Consigna } \\
\text { de localización } \\
\text { y asignatura }\end{array}$ & $\begin{array}{l}\text { Párrafo del texto donde se localiza } \\
\text { la respuesta }\end{array}$ \\
\hline $\begin{array}{l}\text { ¿Qué errores } \\
\text { comete el FMI, } \\
\text { según este } \\
\text { Premio Nobel } \\
\text { [Joseph } \\
\text { Stiglitz]? } \\
\text { (Geografía } \\
\text { Económica) }\end{array}$ & $\begin{array}{l}\text { "El FMI ha cometido errores en todas las áreas en las que ha } \\
\text { incursionado: desarrollo, manejo de crisis y transición del } \\
\text { comunismo al capitalismo. Los programas de ajuste estructural } \\
\text { no aportaron un crecimiento sostenido ni siquiera a los países } \\
\text { que como Bolivia, se plegaron a sus rigores; en muchos países, la } \\
\text { austeridad excesiva ahogó el crecimiento, los programas } \\
\text { económicos que tienen éxito requieren un cuidado extremo en su } \\
\text { secuencia, el orden de las reformas y ritmo." (Manual de Historia } \\
\text { Mundial Contemporánea) }\end{array}$ \\
\hline $\begin{array}{l}\text { Explicar cómo } \\
\text { se formó la } \\
\text { Unión Europea } \\
\text { (Geografía } \\
\text { Económica) }\end{array}$ & $\begin{array}{l}\text { "La Unión Europea (UE) se ha ido construyendo por etapas, a } \\
\text { partir de acuerdos sectoriales (minería, energía), que luego se } \\
\text { fueron unificando al crearse organizaciones supranacionales; } \\
\text { esto es, instituciones que se hallan por encima de los Estados y } \\
\text { que están integradas por sus representantes." (Manual de } \\
\text { Geografía) }\end{array}$ \\
\hline $\begin{array}{l}\text { ¿Cuáles son las } \\
\text { modalidades } \\
\text { básicas de los } \\
\text { paraísos } \\
\text { fiscales? } \\
\text { (Legislación } \\
\text { Fiscal) }\end{array}$ & $\begin{array}{l}\text { "No todos los territorios ofrecen las mismas posibilidades, de allí } \\
\text { que sus clientes pueden optar según sus necesidades específicas. } \\
\text { En función de las diferencias centrales, se pueden establecer tres } \\
\text { modalidades básicas entre ellos: los paraísos fiscales "clásicos"; } \\
\text { los territorios de elevada fiscalidad que ofrecen ventajas fiscales } \\
\text { a personas y entidades que ejercen sus actividades en el } \\
\text { extranjero; finalmente, territorios que disponen de centros de } \\
\text { servicios complejos (en cuanto a las telecomunicaciones, los } \\
\text { bufetes para el asesoramiento a inversores, la disponibilidad de } \\
\text { bases de datos, de conocimientos muy especializados necesarios } \\
\text { para operar en la economía global y en especial, en las finanzas } \\
\text { globales, entre muchos otros)." (Módulo de Cultura Fiscal) }\end{array}$ \\
\hline $\begin{array}{l}\text { En el impuesto } \\
\text { a las ganancias } \\
\text { ¿qué contempla } \\
\text { la ley acerca de } \\
\quad \text { los } \\
\text { contribuyentes? } \\
\text { (Legislación } \\
\quad \text { Fiscal) }\end{array}$ & $\begin{array}{l}\text { "Asimismo, la Ley de Impuesto a las Ganancias establece una } \\
\text { serie de disposiciones especiales que contemplan la situación } \\
\text { personal y familiar de cada trabajador. Las personas que tienen } \\
\text { ganancias por debajo de los valores previstos por la ley, no están } \\
\text { alcanzados por el Impuesto a las Ganancias." (Módulo de } \\
\text { Cultura Fiscal) }\end{array}$ \\
\hline
\end{tabular}

A partir del análisis de los manuales y módulos escolares propuestos para leer, hallamos que las respuestas a las consignas de localización pueden ser fácilmente identificables en estos materiales. Se trata de preguntas que se resuelven con la información disponible en los textos. En su formulación, estas consignas contienen las mismas palabras que los textos, lo que facilita el reconocimiento de las respuestas a través de una correspondencia término a término entre las preguntas y los párrafos del material (Aisenberg, 2004). Esto se asemeja a lo hallado por Rockwell (1995) en cuestionarios que plantean profesores de Ciencias Sociales 
del nivel primario, cuyas preguntas reproducen frases textuales de los libros, facilitando la localización de las respuestas.

En oposición a estas consignas de localización planteadas en Geografía Económica y Legislación Fiscal, gran parte de las consignas propuestas en Estudios Sociales Argentinos apunta a la comprensión del sentido global del texto $y / o$ su relación con otros materiales leídos (Aisenberg, 2004, 2005a, 2010; Carlino, 2005). Estas consignas, a diferencia de las de localización, demandan operaciones cognitivas más complejas: interpretar el texto, releer otros vistos anteriormente y relacionarlos, y elaborar una respuesta escrita producto de ello. La Tabla 2 ilustra ejemplos de estas consignas e indica las acciones que serían necesarias para contestarlas.

Tabla 2. Consignas globales

\begin{tabular}{|c|c|}
\hline Consigna global & Acciones necesarias para responder \\
\hline $\begin{array}{l}\text { ¿Cómo influyen [en } \\
\text { las juventudes] la } \\
\text { historia y la } \\
\text { tecnología? }\end{array}$ & $\begin{array}{l}\text { Comprender cómo el autor, a lo largo de todo el artículo, } \\
\text { describe la influencia de la historia y la tecnología en las } \\
\text { distintas juventudes. (Artículo periodístico "El difícil arte de } \\
\text { asir a la juventud" del sociólogo Mario Margulis, en } \\
\text { cuadernillo) }\end{array}$ \\
\hline $\begin{array}{l}\text { ¿Qué significa } \\
\text { cuando [el autor] } \\
\text { dice que hay que } \\
\text { remitirse a la } \\
\text { ideología, a los } \\
\text { proyectos políticos, } \\
\text { al pasado para } \\
\text { comprender el } \\
\text { presente? }\end{array}$ & $\begin{array}{l}\text { Interpretar, a través de todo el recorte, cómo el autor expone } \\
\text { que los ciudadanos argentinos conciben la historia nacional, } \\
\text { las consecuencias de esto y la propuesta que hace ante ello } \\
\text { (relacionada con comprender las distintas ideologías y } \\
\text { distinciones partidarias en la historia y usar éstas para } \\
\text { entender el presente). (Recorte de la introducción del libro } \\
\text { "Los mitos de la historia argentina" del historiador Felipe } \\
\text { Pigna, en cuadernillo) }\end{array}$ \\
\hline $\begin{array}{l}\text { Desde esa postura } \\
\quad \text { ideológica } \\
\text { [darwinismo social], } \\
\text { ¿cómo se justifica la } \\
\quad \text { pobreza? }\end{array}$ & $\begin{array}{l}\text { Comprender la postura ideológica del "darwinismo social", } \\
\text { desarrollada a lo largo del artículo, y cómo ésta justifica la } \\
\text { pobreza. (Artículo periodístico "Pobres habrá siempre", en } \\
\text { cuadernillo) }\end{array}$ \\
\hline $\begin{array}{l}\text { ¿Por qué motivos } \\
\text { esta propuesta } \\
\text { [proyecto del } \\
\text { ministro de } \\
\text { economía José Ber } \\
\text { Gelbard] es } \\
\text { coherente con un } \\
\text { modelo capitalista } \\
\text { "hacia adentro"? }\end{array}$ & $\begin{array}{l}\text { Comprender el proyecto económico del ministro Gelbard, } \\
\text { descripto en el texto y relacionarlo con el modelo capitalista } \\
\text { "hacia adentro". (Fragmentos del capítulo "Proceso de } \\
\text { acumulación y política económica en Argentina" del } \\
\text { economista Pedro Paz, en cuadernillo) }\end{array}$ \\
\hline
\end{tabular}

Como puede verse en la Tabla 2, las consignas de Estudios Sociales Argentinos requieren que los estudiantes entiendan conceptos o ideas centrales que los autores sostienen a través de todo un texto (por ejemplo, la influencia de la historia y la tecnología en las juventudes, la justificación de la pobreza desde una postura ideológica específica y el proyecto económico de Gelbard), y/o que los relacionen con temas anteriormente trabajados en clase (como el modelo capitalista "hacia adentro"). En cuanto a los textos indicados para leer, ningún párrafo contesta en forma directa estas consignas. Responderlas exige leer el texto en su totalidad, activar conocimientos previos, interpretar los argumentos que los autores utilizan para exponer sus ideas $y$, en ocasiones, releer otros materiales que permitan comprender mejor y ponerlos en relación.

En las entrevistas realizadas, los docentes de estas materias brindan razones por las cuales optan por un tipo u otro de consignas en los cuestionarios. Según las afirmaciones del profesor de Geografía Económica las preguntas de localización adquieren distintos sentidos a lo largo de los trimestres escolares.

Docente Geografía Económica: Uno puede suponer que el pibe [alumno] va a leer pero la intención es [...] yo poder verificarlo [...] a través de un cuestionario [...]. Las preguntas planteadas de ese trabajo del segundo trimestre englobaban a todo lo que habíamos trabajado en clase [...]. Para lo cual tenían que tener la carpeta completa, de manera tal que el hecho de estar [en clase] tuviese algún tipo de valor y el hecho de no venir pero pedir [a los compañeros] lo que se hizo, también [...]. En el tercer trimestre lo que se da es una gran deserción [se ausentan los estudiantes], [...] tenés cinco pibes un día [...]. Es muy difícil pretender una clase expositiva absoluta porque sino [a los alumnos ausentes] le hacés perder el hilo [la progresión de los temas]. [...] Entonces, [doy] trabajos prácticos que tienen que ver con un tema que sí explicamos [...], trabajos pequeños, cortos, con preguntas [...] que son de resolución diaria, [...] te implica la posibilidad de que el pibe tenga herramientas para poder resolverlos.

Este profesor alega distintos motivos por los cuales decide plantear cuestionarios de localización a sus estudiantes. Por un lado, le permiten corroborar que éstos han leído e intentado responder las consignas. De este modo, el cuestionario funcionaría como instrumento de control del cumplimiento de la tarea. Además, el profesor afirma proponer estos cuestionarios para otorgar valor al hecho de asistir a clase y tener completa la carpeta o pedir a los compañeros lo trabajado cuando se ausentan. Por último, el docente opta por este 
tipo de consignas concisas sobre temas ya dados, pensadas para resolverse en la misma clase, para evitar dictar nuevos contenidos en ocasiones de alto ausentismo.

Contrastando con estos motivos, el profesor de Estudios Sociales Argentinos afirma que propone cuestionarios con consignas globales para intentar que los alumnos lean, interpreten y reflexionen sobre los contenidos del texto.

E: ¿Qué actividades das por ejemplo? Esto que yo vi [durante las observaciones], ipreguntas que están en el cuadernillo [de la materia]?

Docente Estudios Sociales Argentinos: Sí, preguntas que están en el cuadernillo [...], las actividades que están armadas. En el texto anterior [se refiere al texto "Los grupos sociales subalternos en la historiografía argentina", no incluido en el cuadernillo] también les hice un par de cuestionarios, [...] cómo aborda el autor el tema, cómo lo plantea [...]. Una lectura más crítica me parece que tenemos que hacer, reflexionar. Lo que quiero lograr es eso [...], intentar que interpreten el texto [...]. Porque a veces lo que pasa es que ellos leen pero [...] por ahí más abajo del texto sigue la explicación. Por eso yo les digo que hagan una lectura general de todo y después... te va a esclarecer un poco la idea [del autor].

Según este profesor, las consignas globales del cuadernillo de Estudios Sociales Argentinos buscan que los estudiantes comprendan la postura del autor y la evalúen. Para que puedan lograrlo, dice alentar a los alumnos a leer la totalidad del texto y evitar su lectura fragmentada.

Estas diferencias que identificamos en los cuestionarios que proponen los docentes de las asignaturas son percibidas por cinco alumnos del curso, quienes califican como "sencillo" responder las consignas de localización de Geografía Económica y Legislación Fiscal, en oposición a contestar las consignas globales de Estudios Sociales Argentinos.

Lorena: [En Geografía Económica] por ejemplo tenemos el libro [se refiere a los manuales] y nos da las preguntas y las buscamos en el libro, ya está. No es una estructura que vos vas armando. Contestás las preguntas y está bien, no las tenés que... [No continúa la frase] o las podés copiar.

E: ¿Qué hacen con [nombra al profesor de Geografía Económica]?

Guadalupe: Y... trabajos, preguntas y entregar en el mismo día siempre.

E: ¿Y cómo son esas preguntas? ¿Te resultan fáciles?

Guadalupe: Más o menos. Depende el día de [nombra al profesor de Geografía Económica].

E: ¿Algunas veces te parecen complicadas las preguntas?

Guadalupe: Sí, pero no mucho, por ahí prestando atención...está como todo en el texto que él dice [indica leer].

E: ¿Y [qué hacen con] [nombra a la profesora de Legislación Fiscal]?

Guadalupe: Nos dicta y nos da preguntas.

E: ¿Y ustedes las responden con eso que tienen dictado?

Guadalupe: Claro, o sino con algo de la carpeta completa.

Fabiana: [En Geografía Económica, las respuestas a las consignas] lo tenemos en el libro, lo tenemos en la carpeta [...]. Así nos sale más sencillo hacerlo [el trabajo práctico].

E: Y cuando ustedes leen [en Estudios Sociales Argentinos] y me dicen que a veces no entienden, ¿por quécreen que no entienden?

Marisa: Y....no sé. Yo a veces leo y no entiendo.

Claudia: [...] algunas cosas de las palabras... lo que está en el libro. Te ponen una pregunta o lo buscás, no lo encontrás y no lo entendés. A mí me pone nerviosa.

E: ¿Las preguntas?

Claudia: Sí. Que yo lo busco y no lo encuentro en el libro. $Y$ [el profesor] te dice "Sí, está ahí" y yo lo busco y no lo encuentro.

Las alumnas Lorena, Guadalupe y Fabiana afirman que las respuestas a cuestionarios de Geografía Económica y Legislación Fiscal son fácilmente localizables en los materiales y que las consignas también pueden contestarse recurriendo a la carpeta. En razón de ello, estas estudiantes sostienen que este tipo de consignas no comporta gran dificultad. Estas apreciaciones de las alumnas pueden relacionarse con el hecho de que las consignas de localización, al solicitar una modalidad de lectura superficial en la que es suficiente identificar y reproducir la información 
pedida (Aisenberg, 2005a, 2010; Benchimol, Carabajal y Larramendy, 2008; Lerner, Aisenberg y Espinoza, 2009), les resultarían fáciles de responder.

En sentido contrario, Marisa y Claudia dicen no poder identificar en los textos de Estudios Sociales Argentino las respuestas a las consignas globales. En efecto, y tal como sostienen, para contestar preguntas globales, no es suficiente "buscar" y "encontrar" la respuesta sino que es necesario leer la totalidad del texto, poner en relación distintos materiales y construir una respuesta con base en lo que se ha logrado interpretar. Por estos motivos, estas consignas suelen presentar mayores dificultades para ser comprendidas y resueltas por los estudiantes (Aisenberg, 2010). Asimismo, observamos que los textos del cuadernillo de esta materia, al ser principalmente materiales de circulación social no destinados a estudiantes, omiten información porque dan por supuesto ciertos conocimientos de los lectores (Lerner, 2001; Lerner y otros, 1997), lo que dificulta que los alumnos comprendan las ideas.

Además de estas diferencias que divisan los estudiantes en las entrevistas, también relatan que son distintas las experiencias escolares que han tenido en relación con estos tipos de consignas. Tal como mostramos a continuación, los alumnos están acostumbrados a responder consignas de localización como las que se asignan en las Geografía Económica y Legislación Fiscal. En cambio, las consignas globales que se indican contestar en Estudios Sociales Argentinos les resultan novedosas, como un elemento no conocido (Rickenmann, 2007) en la situación didáctica.

Carla: En muy pocas [materias] leés.

Lorena: [...] tanto leer [...] o tener que tener que comprender un texto, no lo tenemos [que hacer]. Solamente en esta materia [se refiere a Estudios Sociales Argentinos]. [...] En realidad no estamos acostumbrados [...] a analizar textos.

Yesica: [El docente de Estudios Sociales Argentinos] lo que quiere es como que amplíes mucho [...] las respuestas [...]. Bueno, eso cuesta un poco. Porque nosotros no leemos mucho [...], no estamos acostumbrados a leer [...], me cuesta explicarlo ahí [en el trabajo práctico], así escribiéndolo.

Denise: Lo que pasa es que tampoco estamos, bah, yo por lo menos, acostumbrados [...] a leer, ¿entendés? [...]. Acá [en la escuela] mucho no... como que no leíamos, ni siquiera en Lengua.

Daiana: [El profesor de Estudios Sociales Argentinos] Él sí, nos da a leer un montón, y nosotros no estamos acostumbrados a leer.

E: Claro, ¿ustedes dicen que es el que más les hace leer?

Daiana: Sí.

[...] E: ¿Y [en las Geografía Económica y

Legislación Fiscal] cómo son los trabajos?

Daiana: Tranqui.

E: ¿Tranquilos?

Daiana: No son difíciles.

E: ¿Qué les preguntan por ejemplo?

Daiana: Cosas sobre la materia o del tema que estamos viendo.

E: Y eso digamos, ¿les resulta fácil hacerlo?

Daiana: Sí.

E: Ajá, iy en la escuela siempre les pidieron que escriban estas cosas o les pedían otras cosas distintas?

Daiana: No, siempre.

Daiana comenta que los trabajos prácticos de Geografía Económica y Legislación Fiscal "no son difíciles" y que constan de preguntas acerca de "cosas sobre la materia o del tema que estamos viendo". Respecto de Estudios Sociales Argentinos, coincide con sus compañeras en que en el curso no están "acostumbrados" a realizar las actividades que allí se piden. Las alumnas afirman que esa materia requiere leer textos extensos, a diferencia de otras asignaturas que tienen o han tenido en la escuela. Asimismo, las estudiantes mencionan que en dicha materia tienen que abordar los textos de un modo con el cual no están familiarizados: "comprender", "analizar" y "explicarlo" a través de respuestas en las "que amplíes mucho". Esta forma de abordaje de textos, referida como novedosa por los alumnos, remite a la exigencia de las consignas globales de leer todo el material, activar conocimientos previos, interpretar los argumentos de los autores y construir una respuesta. 


\section{Discusión}

Los resultados muestran que en los cuestionarios que proponen los profesores de las asignaturas indagadas se conforman por consignas de distinto tipo. En dos materias, Geografía Económica y Legislación Fiscal, se plantean consignas de localización para contestar en clase, que los estudiantes consideran sencillas y a las cuales están habituados. Sus respuestas pueden identificarse fácilmente en porciones específicas de los manuales y módulos, a partir de la similitud de términos entre las preguntas y los párrafos. El docente de Geografía Económica sostiene que, a través de estas consignas que suelen formularse respecto a temas ya vistos, controla el cumplimiento de la tarea por parte de los alumnos, otorga importancia el hecho de asistir a clases y tener la carpeta completa, y evita que aquellos estudiantes ausentes se pierdan la presentación de un nuevo tema. Por su parte, el profesor de la Asignatura Estudios Sociales Aregninos, dado que busca que los alumnos interpreten y reflexionen sobre materiales de circulación social incluidos en el cuadernillo de la materia, plantea consignas globales para contestar de forma independiente, que requieren la construcción de ideas globales, explicaciones o relaciones. Los estudiantes dicen no estar acostumbrados a leer de ese modo y refieren dificultades para realizar las actividades asignadas.

Dada la procedencia socioeconómica de los estudiantes, y considerando las trayectorias escolares discontinuas por las que han atravesado, no resulta menor considerar estos resultados. Las consignas de localización, propuestas en forma mayoritaria en este curso de quinto año del nivel secundario, ya que se indican contestar en dos materias analizadas, propondrían a losestudiantes actividades meramente reproductivas. Este tipo de propuestas áulicas, que exigen una sola respuesta considerada correcta y alientan una escritura mecánica, según Miras (2000) son las más usuales en la enseñanza general. En relación con alumnado proveniente de sectores socioeconómicos desfavorecidos, Wells (1987) identifica que se les proponen actividades que recrean los usos del lenguaje escrito en la vida cotidiana (leer un periódico, escribir una solicitud de empleo, completar un formulario o seguir instrucciones para usar un dispositivo), porque se busca prepararlos para las demandas sociales que los esperan cuando finalicen la escolaridad. Siguiendo a estos autores, nos preguntamos por las oportunidades de aprendizaje de contenidos que se fomentan a través de estas consignas mayoritarias que requieren reproducir párrafos. En oposición a la reproducción se encuentran los usos epistémicos del lenguaje que, según Wells (1987), ofrecen a los alumnos oportunidades de situarse como pensadores creativos y críticos. Promover tales usos requiere, entre otras actividades, que se propongan actividades de planificación, relectura y/o revisión de los textos, examen crítico de los materiales de lectura con orientación de los docentes, diálogo sobre los textos leídos o producidos, comparar interpretaciones individuales, etc. (Dysthe, 1996, 2013; Wells, 1990a, 1990b, 1993). Estas actividades potencialmente epistémicas van en línea con los objetivos del área de Ciencias Sociales, que apuntan a la formación de ciudadanos autónomos, reflexivos y críticos de la realidad social en la que están insertos. Aún más, teniendo en cuenta la procedencia socio-económica de los alumnos y su pasaje por experiencias signadas por la desigualdad, no resulta menor que los docentes enseñen contenidos de Ciencias Sociales a la vez que enseñan prácticas como interpretar lo leído, validar lo interpretado, hacerle preguntas a los textos, cuestionar a los autores y construir una postura basada en argumentos. Estas prácticas no forman parte del curriculum de la formación docente en el país, por lo cual, la mayoría de los profesores disciplinares desconocen cómo integrar la lectura y la escritura en la enseñanza de sus asignaturas.

Aquí se señala, entonces, la necesidad de revisar las condiciones de enseñanza que se ofrecen a estos estudiantes y plantear posibles cambios que puedan ser tenidos en cuenta en la formación docente. Deesa forma, se puntúan vías para promover inclusión educativa, ofreciendo educación de calidad para todos los estudiantes, a través de usos de la lectura y la escritura que se han mostrado fructíferos para el aprendizaje de contenidos. 


\section{Referencias bibliográficas}

Aisenberg, B. (2004). Entrar al mundo de un texto. La educación en nuestras manos, 72, 2729.

Aisenberg, B. (2005a). La lectura en la enseñanza de la historia: las consignas del docente y el trabajo intelectual de los alumnos. Lectura y Vida, 26(3), 22-31.

Aisenberg, B. (2005b). Una aproximación a la relación entre la lectura y el aprendizaje de la historia. Revista Íber, 43, 94-104.

Aisenberg, B. (2010). Enseñar Historia en la lectura compartida. Relaciones entre consignas, contenidos y aprendizaje. En I. Siede (Coord.), Ciencias Sociales en la escuela. Criterios y propuestas para la enseñanza (pp. 63-98). Buenos Aires: Aique.

Basuyau, C. y Guyon, S. (1994). Consignes de travail en histoire-géographie: contraintes et libertés. Revue française de pédagogie, 106, 39-46.

Benchimol, K., Carabajal, A., y Larramendy, A. (2008). La lectura de textos históricos en la escuela. Lectura y Vida, 29(1), 22-31.

Carlino, P. (2005). Escribir, leer y aprender en la universidad. Una introducción a la alfabetización académica. Buenos Aires: Fondo de Cultura Económica.

Carlino, P., Roni, C., Rosli, N., Calderón, L., Cordero, G. y Venazo, I. (en prensa). Hablar sobre lo leído para construir conocimiento. Estudios observacionales en Ciencias Sociales y Biología de nivel secundario. Universidad Pedagógica Nacional.

Chevallard, Y. (1991). La transposición didáctica. Del saber sabio al saber enseñado. Buenos Aires: Aique.

Dysthe, O. (1996). The multivoiced classroom: interactions of writing and classroom discourse. Written Communication, 13(3), 385-425.
Dysthe, O. (2013). Perspectivas teóricas sobre el diálogo y la enseñanza basada en el diálogo. En O. Dysthe, N. Bernhardt, y L. Esbjørn, Enseñanza basada en el diálogo. El museo de arte como espacio de aprendizaje (pp. 45-80). Copenhague: Skoletjenesten.

Lerner, D., Levy, H., Lotito, S., Lobello, S., Llorente, E., y Natali, N. (1997). Leer textos difíciles. Documento de trabajo №4. Actualización curricular en Lengua. Ciudad de Buenos Aires: Dirección de Curriculum, Secretaría de Educación. Recuperado de http://www.buenosaires.gob.ar/areas/ educacion/curricula/docum/areas/prleng/ doc4.pdf

Lerner, D. (2001). Leer y escribir en la escuela: lo real, lo posible y lo necesario. México: Fondo de Cultura Económica

Lerner, D., Aisenberg, B., y Espinoza, A. (2009). La lectura en Ciencias Sociales y en Ciencias Naturales: objeto de enseñanza y herramienta de aprendizaje. En J. A. Castorina y V. Orce (Coord.), Anuario del Instituto de Investigaciones en Ciencias de la Educación 2008. Buenos Aires: Editorial de la Facultad de Filosofía y Letras de la Universidad de Buenos Aires. Recuperado de http://www.filo.uba.ar/ contenidos/investigacion/institutos/Iice/ ANUARIO_2008/textos/36_Delia_Lerner. pdf

Miras, M. (2000). La escritura reflexiva. Aprender a escribir y aprender acerca de lo que se escribe. Infancia y Aprendizaje, 89, 65-80.

Rickenmann, R. (2006). Metodologías clínicas de investigación en didácticas y formación del profesorado: un estudio de los dispositivos de formación en alternancia. Actas del Congreso Internacional de Investigación, Educación y Formación docente, Universidad de Antioquia, Medellín, Colombia. Recuperado de http://www.unige.ch/ fapse/clidi/textos/Clinica-did\%E1ctica-RR. pdf 
Rickenmann, R. (2007). Investigación y formación docente: dispositivos de formación $y$ elementos para la construcción de una identidad profesional. EccoS, 9(2), 435463. Recuperado de http://www.uninove. br/PDFs/Publicacoes/eccos/eccos_v9n2/ eccosv9n2_2i14.pdf

Rockwell, E. (1995). En torno al texto: tradiciones docentes y prácticas cotidianas. La escuela cotidiana. México: Fondo de Cultura Económica.

Rosli, N. (2016). Leer y escribir en tres asignaturas de una escuela secundaria a la que asisten alumnos de sectores socioeconómicos desfavorecidos (Tesis doctoral). Doctorado en Ciencias de la Educación. Universidad Nacional de La Plata. Recuperado de http://www. memoria.fahce.unlp.edu.ar/tesis/te.1237/ te.1237.pdf

Rosli, N. y Carlino, P. (2017). Intervenciones docentes en la tarea de leer para responder cuestionarios. Actualidades Investigativas en Educación, 17(3), 1-26.
Rosli, N. y Carlino, P. (En prensa). Puestas en común de respuestas escritas a guías de lectura en materias del nivel secundario. Conocer la escritura: investigación más allá de las fronteras, Pontificia Universidad Javeriana y Writing Across the Curriculum Clearinghouse.

Wells, G. (1987). Apprenticeship in literacy. Interchange, 18(1/2), 109-123.

Wells, G. (1990a). Creating the conditions to encourage literate thinking. Educational Leadership, 47(6), 13-17.

Wells, G. (1990b). Talk about text: where literacy is learned and taught. Curriculum Inquiry, 20(4), 369-405.

Wells, G. (1993). Text, talk and inquiry: schooling as semiotic apprenticeship. International Conference on Language and Content, Hong Kong. 


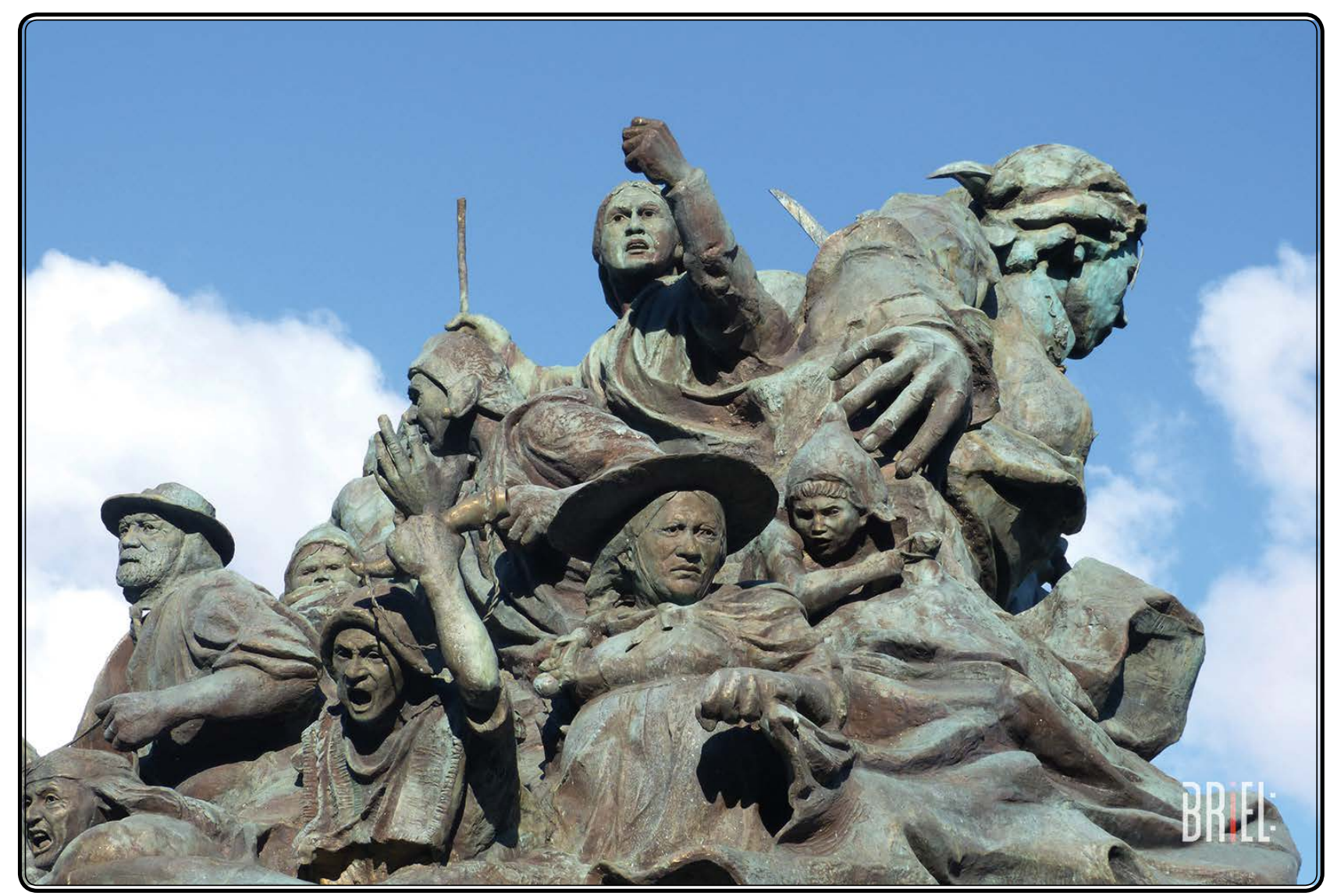

Los de abajo, borrados pero en resistencia. 